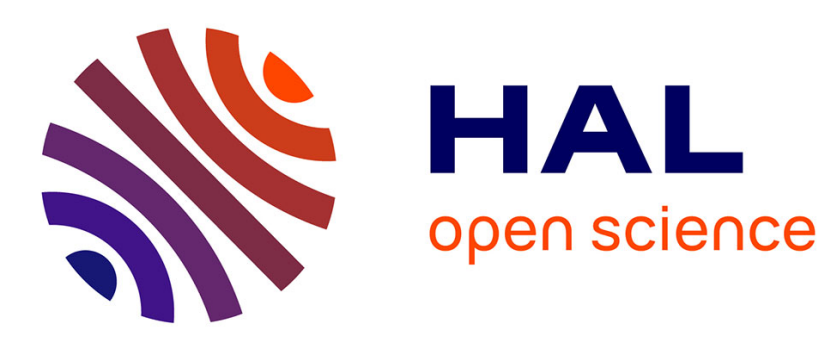

\title{
Intracellular mechanics: connecting rheology and mechanotransduction
}

\author{
Samuel Mathieu, Jean-Baptiste Manneville
}

\section{To cite this version:}

Samuel Mathieu, Jean-Baptiste Manneville. Intracellular mechanics: connecting rheology and mechanotransduction. Current Opinion in Cell Biology, 2019, 56, pp.34-44. 10.1016/j.ceb.2018.08.007 . hal-01955735

\section{HAL Id: hal-01955735 \\ https://hal.sorbonne-universite.fr/hal-01955735}

Submitted on 14 Dec 2018

HAL is a multi-disciplinary open access archive for the deposit and dissemination of scientific research documents, whether they are published or not. The documents may come from teaching and research institutions in France or abroad, or from public or private research centers.
L'archive ouverte pluridisciplinaire HAL, est destinée au dépôt et à la diffusion de documents scientifiques de niveau recherche, publiés ou non, émanant des établissements d'enseignement et de recherche français ou étrangers, des laboratoires publics ou privés. 


\section{Intracellular mechanics: connecting rheology and mechanotransduction Samuel Mathieu ${ }^{1,2}$ and Jean-Baptiste Manneville ${ }^{1,2}$}

Cell mechanics is crucial for a wide range of cell functions, including proliferation, polarity, migration and differentiation. Cells sense external physical cues and translate them into a cellular response. While force sensing occurs in the vicinity of the plasma membrane, forces can reach deep in the cell interior and to the nucleus. We review here the recent developments in the field of intracellular mechanics. We focus first on intracellular rheology, the study of the mechanical properties of the cell interior, and recapitulate the contribution of active mechanisms, the cytoskeleton and intracellular organelles to cell rheology. We then discuss how forces are transmitted inside the cell during mechanotransduction events, through direct force transmission and biochemical signaling, and how intracellular rheology and mechanotransduction are connected.

\footnotetext{
Addresses

${ }^{1}$ Institut Curie, PSL Research University, CNRS, UMR 144, 26 rue d'UIm, F-75005, Paris, France

${ }^{2}$ Sorbonne Université, UPMC University Paris 06, CNRS, UMR 144, 26 rue d'Ulm, F-75005, Paris, France
}

Corresponding author: Manneville, Jean-Baptiste (Jean-Baptiste. Manneville@curie.fr)

\section{Introduction}

Cells interact physically with their environment. They can probe the mechanical properties of their substrate and apply forces onto their surroundings. Conversely, external mechanical constraints can induce cellular responses. In a process called mechanotransduction [1,2], external forces, in the form of tensile, compressive or shear stresses, are sensed by cells and transmitted to the cell interior to elicit a new cell behavior. Force transmission within the cell interior may be affected by the mechanical properties of the cytoplasm. Intracellular rheology, which focuses specifically on the mechanical properties of the cell interior, has received a growing interest in recent years [3,4]. The general view is that the cell interior behaves as a viscoelastic material and its frequency-dependent rheological moduli follow a weak power law [5]. However, whether intracellular material properties can impact on mechanotransduction and reciprocally is still unclear.

In this review, we first discuss the latest findings in intracellular rheology with an emphasis on the critical role of the spatial and temporal scales. The contribution of active out-of-equilibrium forces has recently taken center stage. We also describe how the cytoskeleton [3] and intracellular organelles participate to intracellular rheology. We then turn to mechanotransduction and how forces are transmitted from the plasma membrane to the cell interior and intracellular organelles. Here again, spatio-temporal scales appear to be crucial to determine whether forces are directly transmitted to organelles via a purely physical mechanism or whether biochemical signaling is required. Throughout this review, we chose to highlight recent studies in which quantitative mechanical measurements were performed, mostly in mammalian cells. We do not detail the latest technical developments in the field of intracellular mechanics and refer to Refs. [6-12] for recent reviews on the technical aspects.

\section{Intracellular rheology Mechanics of the cell cytoplasm and role of non- equilibrium active forces}

Because most cellular functions are regulated by intracellular processes that take place in the cytoplasm, intracellular rheology has emerged as an essential aspect of cell mechanics. While the cytoplasm can be viewed as a viscoelastic material, its mechanical properties strongly depend on the spatio-temporal scale at which they are probed. First, the size of the probe used to measure intracellular viscoelastic moduli determine which cytoplasmic structures will dominate the measurement (Figure 1a). The cytoplasm was shown to behave as an elastic solid using 100-500 nm-size optically-trapped beads [13] or as a viscoelastic liquid using micron-size superparamagnetic wires [14]. Second, the cytoplasm is not homogeneous and its rheology depends on the position within the cell. The perinuclear region is usually stiffer than the cell periphery $\left[15^{\circ}\right]$. This may reflect differences in cytoplasmic crowding, for instance in regions of high densities of cytoskeletal or soluble proteins. Consistently, when the cell volume decreases, intracellular stiffness increases $\left[16^{\bullet}\right]$. Third, the viscoelastic properties of the cytoplasm are strongly frequencydependent $\left[17^{\bullet \bullet}\right]$. 


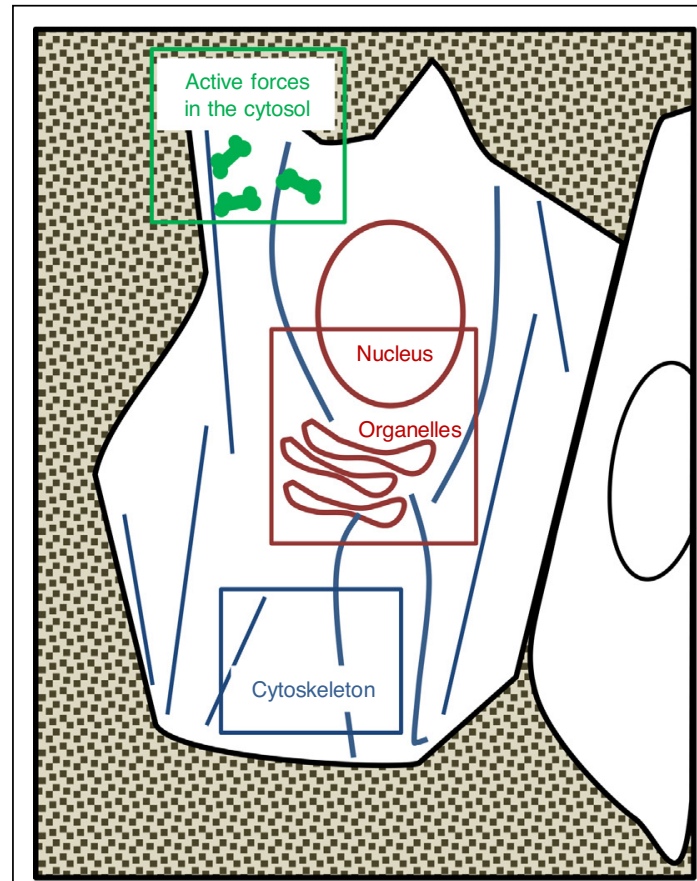

(a) - Active forces in the cytosol
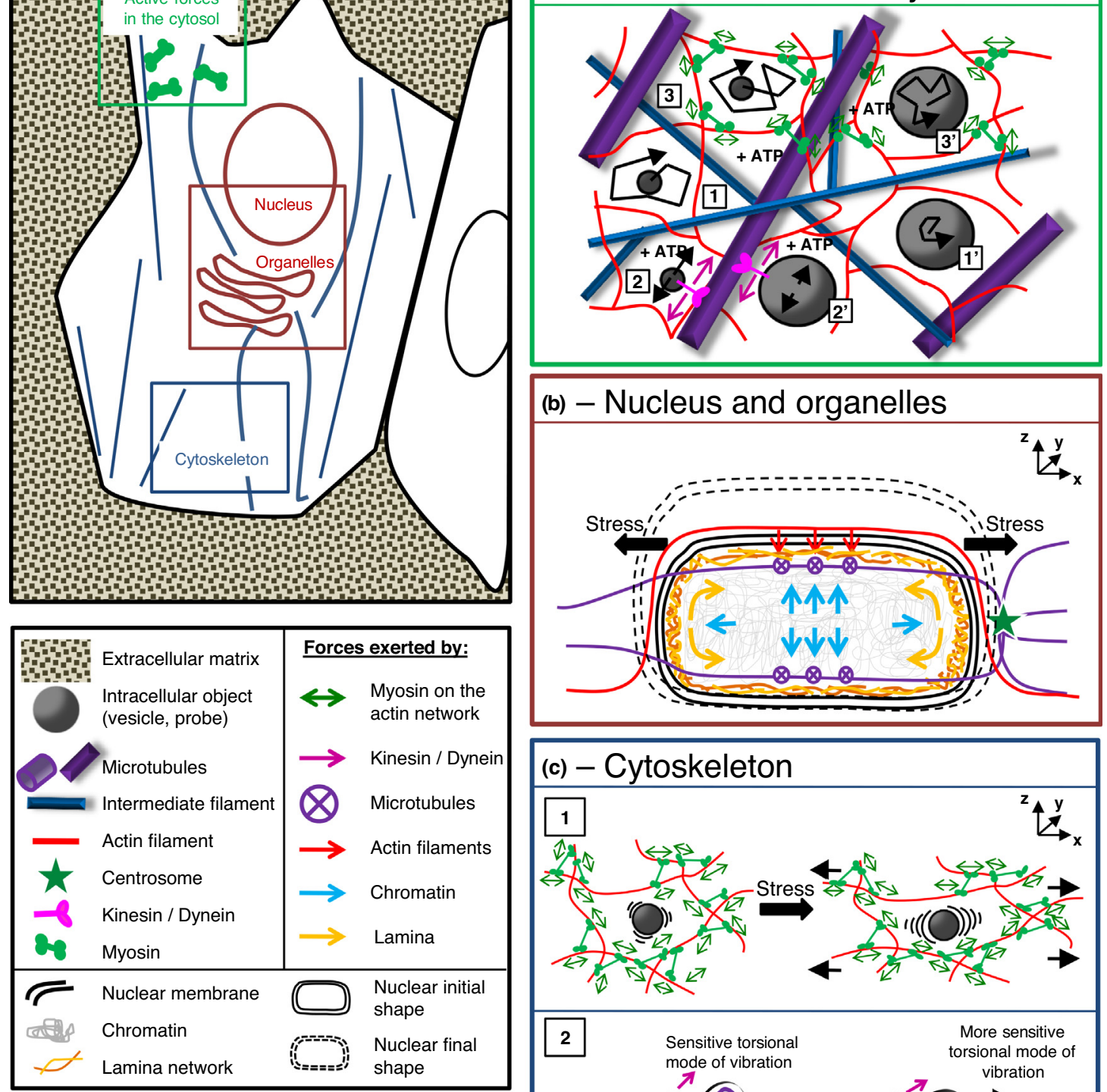

(b) - Nucleus and organelles
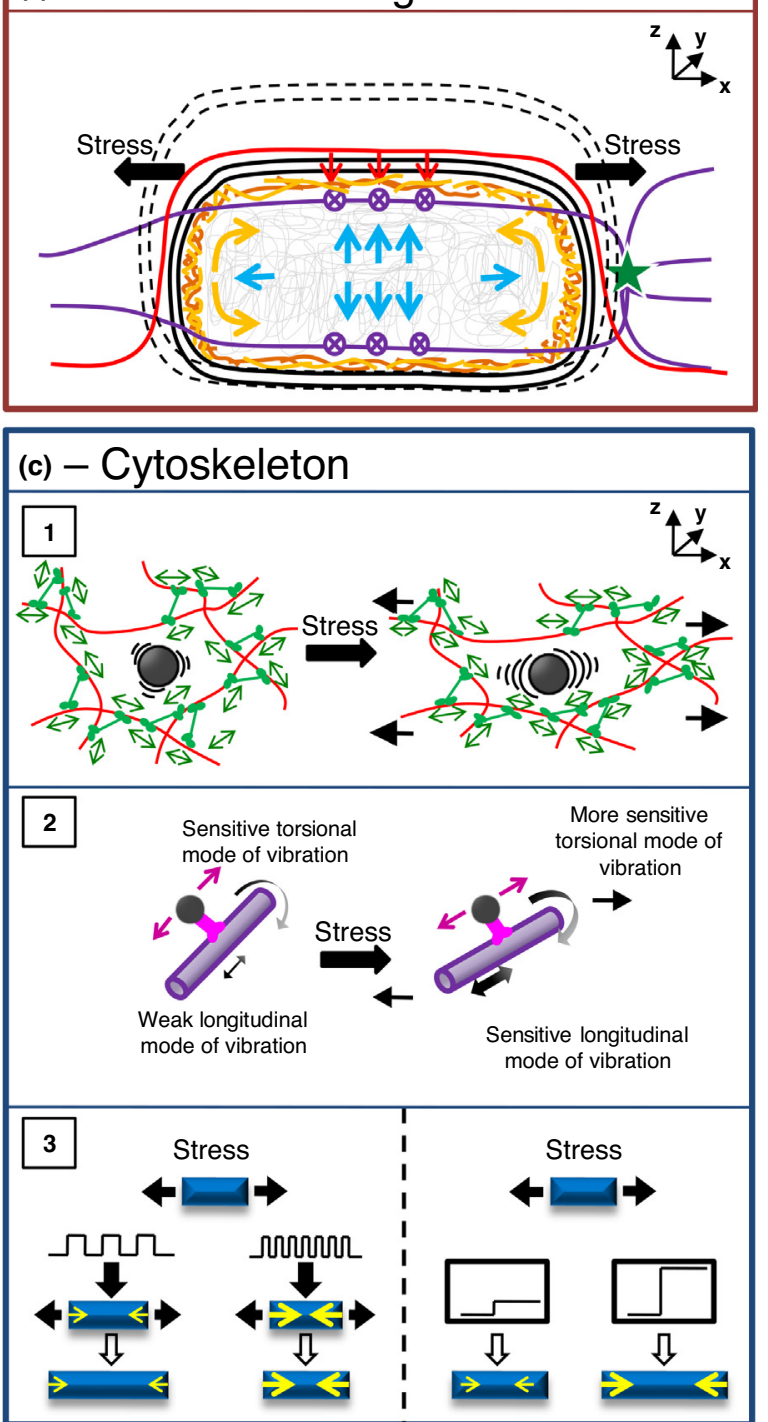

Some examples of recent advances in intracellular rheology.

(a) Active forces in the cytosol. The mechanics of the cytoplasm depends on the frequency at which it is probed and on the size of the probe. 
(Figure 1 Legend Continued) Thermal equilibrium random forces (1 and 1') dominate the high frequency regime (short time scales), while active non-equilibrium ATP-dependent forces generated by molecular motors (2 and 2': kinesins and dynein; 3 and 3': myosins) dominate the low frequency regime (long time scales). Myosin motors exert active forces on the actin network which are transferred to the probes (3 and $\left.3^{\prime}\right)$. Small probes (1, 2, 3, typically $<100 \mathrm{~nm}$ in diameter) are less prone to frictional forces and move more easily than larger probes (1', 2', 3', typically $>$ $1 \mu \mathrm{m}$ in diameter). As a consequence, in active rheology experiments, higher forces have to be applied to larger probes. Depending on the probe size, different intracellular elements contribute to the measurement.

(b) Auxetic properties of the nucleus. The shape of the nucleus is regulated by a balance between forces exerted by the actin cap, microtubules and resistance to deformation provided by chromatin and the lamina network. A stretch applied to the nucleus (large black arrows, in the xdirection) could induce a de-condensation of chromatin along the stress axis (horizontal blue arrows) and an increase in lamina tension at the poles of the nucleus (orange arrows) which reinforces the lamina in these regions. Away from the poles, lamina tension relaxes and chromatin decondensation occurs perpendicularly to the stress direction (vertical blue arrows). Together with the balance of forces exerted by actin (red arrows, in the minus z-direction) and microtubules (purple symbols, in the plus or minus y-direction), these events may lead to auxetic properties, i.e. thickening (instead of thinning) of the nucleus in the direction perpendicular to the applied stretch. The solid and dashed black lines represent the shape of the nucleus before and after application of the stress respectively.

(c) Cytoskeleton mechanical properties and roles in intracellular mechanics. The actomyosin network (1) exerts forces on intracellular objects, provides resistance to intracellular stresses, and, when stretched, induces local tension and increases cytoplasm rigidity. Models show that microtubules (2) could be more susceptible to torsional modes of vibration than to longitudinal modes of vibration. When microtubules are stretched, vibration modes can become more sensitive to model parameters and increase both translation and rotation velocities of vesicles along the filaments. Vibration modes can also play a role in regulating the rheological parameters of the cytoplasm. Intermediate filaments (3) exhibit a mechanical response that strongly depends on the loading rate. A slowly applied stress or a small deformation efficiently deforms intermediate filaments, while a stress applied at a high frequency or a large deformation reinforces their mechanical resistance.

That spatio-temporal scales are critical for intracellular rheology is the signature of non-equilibrium active forces within the cell. Because a living cell consumes energy, for instance in the form of ATP, from a statistical physics point of view, it is out-of-equilibrium. The cytoplasm is thus subjected to thermal (equilibrium) noise and to active (non-equilibrium) noise originating mostly from the cytoskeleton and its associated motors [13] (Figure 1A). The three types of cytoskeletal fibers, actin, microtubules and intermediate filaments, all contribute to intracellular rheology. However their roles vary with the cell type. In general polymerized actin stiffens the cytoplasm, as shown in adherent mammalian cells $\left[15^{\circ}, 18^{\circ}\right]$ or in mouse oocytes using ultrafast optical microelastography $\left[19^{\bullet \bullet}\right]$. Reducing actin cross-linking by inhibiting Arp $2 / 3$ reduces the elastic and viscous moduli at low frequencies $\left[18^{\circ}\right]$. In contrast, the actin nucleator formin-2 does not contribute to intracellular stiffness in oocytes $\left[17^{\bullet \bullet}\right]$. Depolymerizing microtubules softens the cytoplasm of adherent mammalian cells [15 $\left.{ }^{\circ}\right]$ but has no effect in oocytes cells $\left[17^{\bullet \bullet}\right]$. Finally, intermediate filament proteins also contribute to intracellular rheology, as shown for vimentin in mouse embryonic fibroblasts [20] and more recently for desmin in myoblasts [ $\left.21^{\circ}\right]$.

Actomyosin contractility and myosin motors are major regulators of intracellular rheology. Upon inhibition of myosin II, the elasticity of the cytoplasm significantly decreases at long timescales whereas its viscosity strongly increases at short timescales [ $\left.18^{\circ}\right]$. When myosin $\mathrm{V}$ activity is inhibited in oocytes, the cytoplasm stiffens indicating increased cross-linking $\left[17^{\circ}\right]$. Active ATP-dependent processes not only induce short range fluctuating displacements that dominate over thermal noise at low fre-

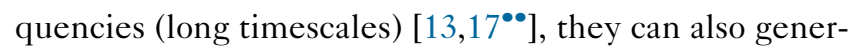
ate large scale motions within the cytoplasm. Such activity-driven cytoplasmic flows, such as the actin retrograde flow, impact on intracellular organization and can reposition intracellular organelles or reorient intracellular trafficking. In the Caenorhabditis elegans zygote, cytoplasmic flows are thought to be generated by an actomyosin-dependent cortical flow which polarizes the zygote during the first asymmetric cell division. By artificially inducing cytoplasmic flows, a strong coupling between cytoplasmic and cortical flows was recently shown to induce the polarization of membrane-bound partition-

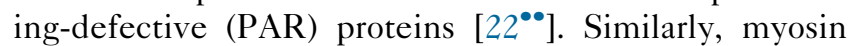
$\mathrm{V}$ regulates the diffusion of actin-coated vesicles and generates a pressure gradient responsible for nucleus centering in mouse oocytes [23].

\section{Mechanics of intracellular organelles}

In most cell types, intracellular organelles occupy more than half the volume of the cytoplasm and are thus major determinants of intracellular mechanics. The nucleus, the largest organelle, is obviously the most studied. From a mechanical point of view, the nucleus is a three-layer assembly of macromolecules (chromatin) surrounded by a protein network (the nuclear lamina, composed of the intermediate filament proteins of the lamin family) and a double membrane (the nuclear envelope). Each layer contributes to nuclear mechanics (see Refs. [24,25] for reviews). The unique mechanical properties of the nucleus arises primarily from the combined effects of chromatin condensation and the network organization of the lamina [26-28]. On short timescales and for stresses similar to those that cells can generate through cytoskeletal forces, nuclei display a fluid-like rheology probably dominated by chromatin, whereas under sustained stresses the nucleus behaves more like a solid due to the lamina [26]. Nuclei of lamin-deficient cells exhibit a more fluid-like behavior, especially at short timescales [26]. More recently, nuclear deformations were shown to correlate with stiffening events using a technique allowing side-view imaging combined with AFM indentation $\left[29^{\circ}\right.$ ]. Micromanipulation of isolated nuclei has revealed 
firstly that the nucleus can adjust its stiffness on short time scales (less than $1 \mathrm{~min}$ ) and stiffens in response to an oscillatory stress $[30,31]$, and secondly that chromatin is the main regulator of mechanical responses to small (few $\mu \mathrm{m})$ deformations, while lamin $\mathrm{A} / \mathrm{C}$ levels modulate nuclear stiffening against larger extensions [32 $\left.2^{\bullet \bullet}\right]$. These studies suggest that chromatin condensation/de-condensation events may occur at short timescales in a reversible manner and that the lamina induces nuclear stiffening to prevent DNA damage against strong deformations. Intriguingly, temperature seems to impact strongly on the material properties of the nucleus in a way that depends on chromatin compaction and remodeling dynamics $\left[33^{\circ}\right]$.

Cytoskeletal networks participate actively in nuclear mechanics. In cardiac myocytes for instance, actin stiffens the nucleus while microtubules have the opposite effect [34]. However the effects of the cytoskeleton on nuclear stiffness probably depend on the cell type since recent results demonstrate that both actin and microtubules stiffen the nucleus and slow down the viscoelastic relaxation of the nucleus in human bladder cancer cells [ $\left.35^{\circ}\right]$. The role of the cytoskeleton in nuclear mechanics is particularly evident when cells have to migrate in a confined environment, for instance through constrictions or narrow pores, during which the nucleus has to squeeze and the nuclear envelope may rupture. A recent model of cells transmigrating through pores which includes actomyosin contractility and cytosolic back pressure predicts thresholds for lamina buckling and rupture [36 ${ }^{\circ}$. The cytoskeleton also confers auxetic properties to the nucleus: when stretched, the nucleus becomes thicker in the direction perpendicular to the applied force, as opposed to classical materials [37 $7^{\circ \bullet}$ ] (Figure 1b). These studies suggest that when cells migrate through narrow constrictions, the cytoskeleton both helps the nucleus to release and redistribute the mechanical load, with actin providing local stiffening and microtubules inducing softening along the nucleus major axis, and protects the nucleus against extreme deformations by controlling nuclear envelope rupture.

The nucleus is not the only organelle which mechanics may be critical for cellular processes. The mechanics of the Golgi apparatus [38,39] for instance could play a major role in intracellular trafficking. Mechanically, the Golgi cisternae, like the nucleus, can be thought of as a threelayer system: Golgi membranes, a matrix of coiled-coiled proteins called the Golgi matrix and the surrounding cytoskeleton. Using optically-trapped beads, forces were applied to Rab6-positive Golgi cisternae and the viscoelastic properties of these cisternae were measured [40]. It was shown that actomyosin contractility and the Golgi matrix protein giantin both stiffen the Golgi apparatus and that a local force delays myosin II-dependent fission of Rab6-positive transport carriers.

\section{Mechanics of the cytoskeleton}

While the cytoskeleton is clearly involved in the mechanics of the cytoplasm and of intracellular organelles, its own intrinsic mechanical properties are also crucial for intracellular rheology (Figure 1c). Quantitative measurements of the mechanical properties of cytoskeletal fibers have mostly been performed using in vitro reconstitution assays of actin, microtubule or intermediate filament networks [3]. For instance, in vitro reconstituted intermediate filament networks exhibit fascinating mechanical properties characterized by a stress-stiffening nonlinear elasticity [41]. Recently, the stress-strain curves of single vimentin intermediate filaments have been measured by optical tweezers and AFM experiments [ $\left.42^{\circ}\right]$. The results show that the mechanical response of vimentin filaments is non-linear and depends strongly on the loading rate. Vimentin filaments act as 'safety-belts': they are soft during a slow deformation, and stiffen under a fast deformation [42]. The mechanical properties of the cytoskeleton can also be measured or predicted from numerical and theoretical models. For instance, structural mechanics models suggest that microtubules resist tension efficiently but are more susceptible to torsion [43] and that torsional modes of vibration are more sensitive to model parameters, such as the cytosol viscosity, than longitudinal modes [44]. Even if in vitro, in silico or theoretical results provide extremely valuable information, they have to be validated by experiments in living cells. Direct in cellulo measurements of the mechanical properties of cytoskeletal networks are still scarce. The observation of the buckling of keratin intermediate filament bundles in living cells allowed estimating their viscoelastic properties [45]. More recently, the viscoelastic properties of single actin stress fibers were measured in cells perforated by microsurgery [46]. However, extensive measurements of the rheology of individual or bundled actin fibers, microtubules and intermediate filaments in living cells are still lacking. Super resolution microscopy combined with mechanical measurements should also help deciphering how the three cytoskeletons mechanically interact.

\section{Intracellular mechanotransduction}

Mechanotransduction is the process by which external forces are sensed by the cell, transmitted to the cell interior and converted into a cellular response. It is now well accepted that external forces can reach far into the cell and are sensed by intracellular organelles. Several mechanosensitive modules, that is protein complexes that sense and respond to forces, have been identified $[1,24]$. At the plasma membrane, focal adhesions between the cell and the extracellular matrix (ECM), cell-cell junctions and stretch-activated ion channels are the best characterized mechanosensitive modules. Inside the cell, the nucleus is the main mechanosensitive organelle identified so far, owing to its associated LINC (Linker of Nucleoskeleton and Cytoskeleton) complex. These 
mechanosensitive modules are linked together by cytoskeletal fibers, suggesting that forces could be transmitted directly inside the cell (Figure 2). The molecular machinery responsible for mechanotransduction has been characterized at a growing pace in recent years (see Refs. [4751] for recent reviews). Below, rather than molecular mechanisms, we focus more on the physical aspects of mechanotransduction and on quantitative measurements using novel methodologies.

\section{Direct force transmission versus biochemical signaling} How is a force transduced by a cell from the extracellular environment to the cell interior? To answer this question, different mechanisms have been proposed involving direct force transmission and biochemical signaling, depending on the spatial localization of the mechanosensitive module within the cell and on the time scale at which mechanotransduction and subsequent cell responses occur (Figure 2). Effective mechanotransduction involves three consecutive steps: firstly force sensing, secondly force transduction, and thirdly an intracellular response. These three steps do not necessarily happen at the same subcellular localization and have to be tightly regulated in time and space.

The first mechanism is based on biochemical signaling (Figure 2a). Because they rely mostly on diffusion, mechanisms based on biochemical signaling and their associated cell responses are slow, typically on the time scales of minutes to hours. The second mechanism is based on mechanics and direct force transmission by the cytoskeleton and molecular motors (Figure 2b). Because force transmission is achieved by purely physical mechanisms, mechanotransduction and cell responses can be much faster than through biochemical signaling (typically on the time scale of a few seconds or shorter). Such mechanisms rely heavily on the cytoskeleton, motor activity and cell contractility, which are energy-consuming processes. ATP-dependent out of equilibrium activity is thus required for efficient mechanotransduction based on direct force transmission. The third mechanism includes both biochemical signaling and direct force transmission, and as such is probably the most likely scenario (Figure 2c).

\section{Mechanosensing at focal adhesions and cell-cell junctions}

Focal adhesions and cell-cell junctions are the two best described mechanosensitive modules [47-50]. Through these modules, cells can sense and adapt to the mechanical properties of their substrate, their surrounding environment or neighboring cells. The intracellular mechanisms of force transmission and associated signaling pathways at focal adhesions and cell-cell adhesions are currently under intense investigation, and the so-called molecular clutch hypothesis, which relies on cell contractility, actin retrograde flow and the presence of at least two molecular force sensors in series, emerges as a main driver of mechanotransduction at focal adhesions [48].

Among the numerous proteins composing focal adhesion complexes, integrin, talin, tensin and vinculin play key roles in mechanotransduction [47,52]. FRET-based assays have allowed dissecting the way focal adhesions sense external forces at the molecular level [53 $\left.3^{\circ}\right]$. Consistent with the molecular clutch model, these results suggest that tension in vinculin increases as it treadmills from the proximal to the distal end of the focal adhesion until vinculin detaches from the distal end and is carried back to the proximal end by the actin retrograde flow $\left[53^{\circ}\right]$.

Sensing of substrate rigidity by focal adhesions has been studied in details for many years. Recently, a mechanotransduction signaling pathway involving actomyosin contractility, focal adhesion kinase FAK and Yes-associated protein YAP has been deciphered in ovarian cancer cells $\left[54^{\circ}\right]$. In the context of cancer again, the elastic modulus of bladder cancer cells was shown to increase with substrate rigidity $\left[55^{\circ}\right]$. A novel signaling pathway in cell-substrate adhesion-induced mechanotransduction was recently discovered and involves the metabolic sensor AMP-activated protein kinase (AMPK) $\left[56^{\circ}\right]$. Here again, integrin-dependent signaling at focal adhesions during mechanotransduction appears to impact on intracellular rheology.

Similar to focal adhesions, cell-cell junctions are mechanosensitive modules. Adherens junctions are well-known mechanosensors through its coupling to the actin cytoskeleton (see Refs. [47,50] for reviews). Less characterized is the response of desmosomes to mechanical forces [57]. The mechanical link between desmosomes and intermediate filaments through desmoplakin has been identified as a potential force transducer module [58]. However, the effects of the connection between desmosomes and intermediate filaments depend on the actin cytoskeleton and on actomyosin contractility, suggesting that a balance of forces between the different cytoskeletal systems is critical in mechanotransduction at cell-cell junctions.

\section{Force transmission to intracellular organelles}

Once the external force has been sensed at the plasma membrane, the mechanical signal can be transmitted to the cell interior by cytoskeletal fibers as far as the cell nucleus. Because the actin, microtubule and intermediate filament systems are tightly linked and coordinated, when a directional constraint is applied to the cell, for instance by shear stress in endothelial cells under flow [59], the three systems align in the direction of the constraint. However, recent work in muscle cells shows that the direction of newly formed actin stress fibers following a mechanical stretch depends on the cell geometry [60]. 


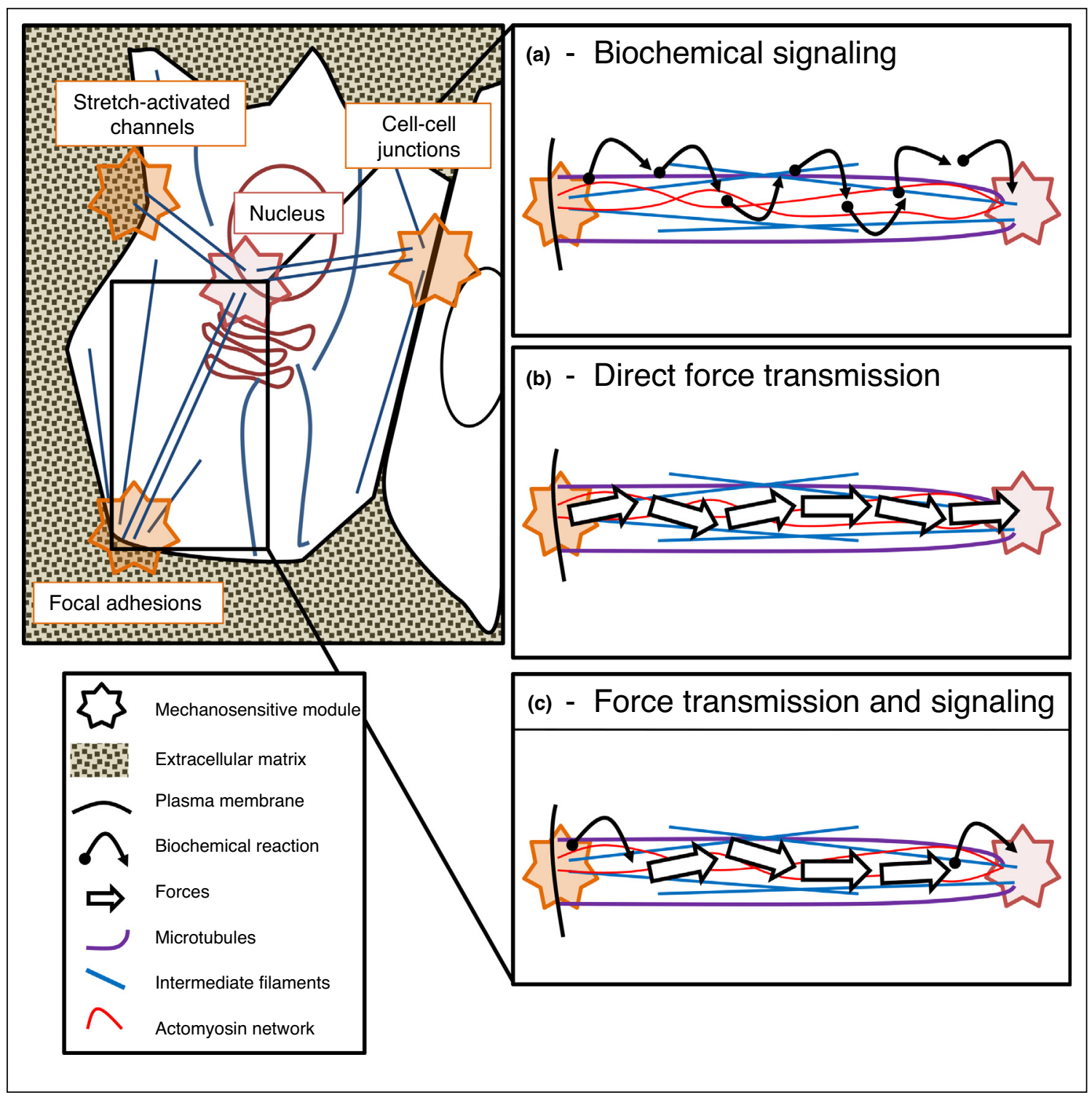

Mechanisms of intracellular mechanotransduction.

(a) Biochemical signaling. An external mechanical cue is sensed at the plasma membrane by a mechanosensitive module and induces a cascade of biochemical reactions (phosphorylation, activation of $\mathrm{G}$ proteins, activation of various signaling pathways) that reach an intracellular target to trigger a cell response (proliferation, migration, gene expression . . . ). This mechanism is more relevant to transduce long lasting external forces inducing a small and localized cellular deformation.

(b) Direct force transmission. The external mechanical cue is sensed at the plasma membrane by a mechanosensitive module directly linked to one or several intracellular mechanosensitive modules. For example, the external force can be sensed through the membrane by integrins at focal adhesions or by cadherins at cell-cell junctions. Direct connections between focal adhesions or cell-cell junctions and the actin cytoskeleton transmit the force along actin fibers towards a mechanosensitive intracellular target, for instance the nucleus. This mechanism can occur on short time scales (seconds) and is more likely to be involved in the case of forces inducing rapid, large and global cellular deformations.

(c) Force transmission and signaling. While force sensing still primarily occurs at the plasma membrane and is transmitted to one or several intracellular mechanosensitive modules mechanically linked together by the cytoskeleton, biochemical signaling also regulates force transduction. Signaling reactions can be spatially localized at various subcellular locations close to the corresponding mechanosensitive module, thus restricting diffusion and allowing faster cellular responses. This mechanism enables the cell to sense and react to a variety of forces that can differ on both spatial and temporal scales.

The direct mechanical link between the plasma membrane and the nucleus is particularly well described (see
Refs. [49,51,61-64] for reviews). Actin and intermediate filaments bridge adhesions at the plasma membrane to 
the LINC complex (SUN and KASH domain proteins, nesprins) and lamin proteins at the nuclear envelope. The mechanical role of this molecular machinery was investigated using single isolated nuclei and optical tweezers [65]. The connection between actin and nesprin was shown to depend not only on the force amplitude but also on the force direction and on the culture conditions. Actomyosin filaments form a perinuclear contractile actin cap above the nucleus in adherent cells [66] which stiffens the nucleus and decreases chromatin dynamics in cells plated on elongated micropatterns [67]. The tension generated by the actin cap and its coupling to the nuclear lamina and the nucleoplasm via the LINC complex also induce a vertical polarization of lamins at the nuclear envelope and of transcriptionally active chromatins towards the apical pole of the nucleus [68]. Several lines of evidence suggest that external forces can be directly transmitted to the nucleus. Upon an osmotic shock, intranuclear structures can remodel on short time scales (less than $20 \mathrm{~s}$ ) and chromatin condensation (resp. decondensation) increases in hyperosmotic (resp. hypoosmotic) conditions [69]. Additionally, in line with the auxetic properties mentioned above, applying forces on whole cells induces a fast (less than $1 \mathrm{~s}$ ) anisotropic deformation of the nucleus preferentially along the short axis of the nucleus $\left[70^{\circ}\right]$, Further proof of a direct force transmission from the extracellular matrix to the nucleus comes from experiments showing that external forces can impact nuclear entry $\left[71^{\circ \bullet}\right]$. When cells are plated on stiff substrates, YAP enters the nucleus [72]. The new results demonstrate that the forces induced by adhesion on stiff substrates, but also by direct indentation with an AFM tip or by osmotic shocks, stretch the nucleus and nuclear pores favoring the active transport of YAP into the nucleus $\left[71^{\circ}\right]$.

While it is now well documented that forces can be directly transmitted from the plasma membrane to the nucleus via the cytoskeleton, it is still not clear whether the same holds for other intracellular organelles. Because vesicular transport in neurons is affected by a mechanical stretch [73] and fission of post-Golgi transport carriers is delayed by a force applied on the Golgi apparatus [40], it is tempting to speculate that the Golgi apparatus is also a mechanosensitive organelle which can sense forces transmitted to the cell interior. Similarly, the endoplasmic reticulum (ER) could bear mechanosensitive properties, since a force applied by optical tweezers at the plasma membrane is transmitted to the ER where it triggers $\mathrm{Ca}^{2+}$ release [74]. Mechanosensitivity is thus probably not restricted to the nucleus and may be a general property of intracellular organelles.

\section{Intracellular responses to external forces}

Mechanical signals transmitted to the cell interior induce a variety of cell responses, including cell differentiation, proliferation, polarity, migration and can affect gene regulation and transcription. It is generally assumed that these responses occur through biochemical signaling downstream of the nucleus. However several lines of evidence suggest that purely physical mechanisms can also directly contribute to the cell responses. First, the nucleus can be bypassed for cell migration in $1 \mathrm{D}$ or $2 \mathrm{D}$ $\left[75^{\circ}\right.$ ]. Enucleated cells, also called cytoplasts, are able to polarize and migrate as single cells in chemotactic or haptotactic assays and collectively in a wound healing assay in 1D and 2D. However, the nucleus is required for migration in $3 \mathrm{D}$ and for cellular responses to substrate rigidity. Second and perhaps more strikingly, a force transmitted from the plasma membrane to the nucleus can have a direct effect on gene transcription $\left[1,76^{\circ \bullet}\right]$. Forces applied to the cell surface induce stretching of the chromatin in the nucleus and a rapid (within $15 \mathrm{~s}$ ) increase in gene expression, favoring a mechanism based on chromatin deformation rather than based on biochemical signaling (Figure 2c).

\section{Concluding remarks and open questions}

Cell rheology and mechanotransduction have been considered as two separate fields. Cell rheology has been most often studied from a physical perspective, while mechanotransduction has attracted the interest of cell biologists. In recent years, several studies have shown that both fields share common features and can impact on each other (Figure 3) [1]. One such common theme is the critical importance of out-of-equilibrium active processes, among which actomyosin contractility, which can regulate intracellular rheology and are at the core of the transduction of a mechanical force into a cellular response. The rheological properties of the cell interior can modulate intracellular force transmission and influence mechanotransduction. Intuitively, a more visco-elastic interior could damp force transmission by slowing down characteristic mechanical relaxation timescales, diffusion of signaling molecules and/or biochemical reactions. Conversely, biochemical signaling pathways can modify the cell viscoelastic properties, not only at the plasma membrane but also at intracellular organelles and the nucleus and in the cytoplasm $\left[55^{\circ}, 56^{\bullet}, 58,67,77,78^{\circ}\right]$. The mechanisms which allow reciprocal interactions between intracellular rheology and mechanotransduction still have to be clarified. For instance, by exerting friction forces on intracellular components, cytoplasmic flows such as the actin retrograde flow could be a major player in intracellular rheology and force transduction and could regulate cell responses, especially those depending on a molecular clutch such as cell adhesion and migration or during immune responses $\left[53^{\bullet}, 79,80\right]$.

For both intracellular rheology and intracellular mechanotransduction, time scales are critical. Rheological moduli strongly depend on the frequency of the mechanical perturbation and intracellular materials usually stiffen with increasing strain. During mechanotransduction, 


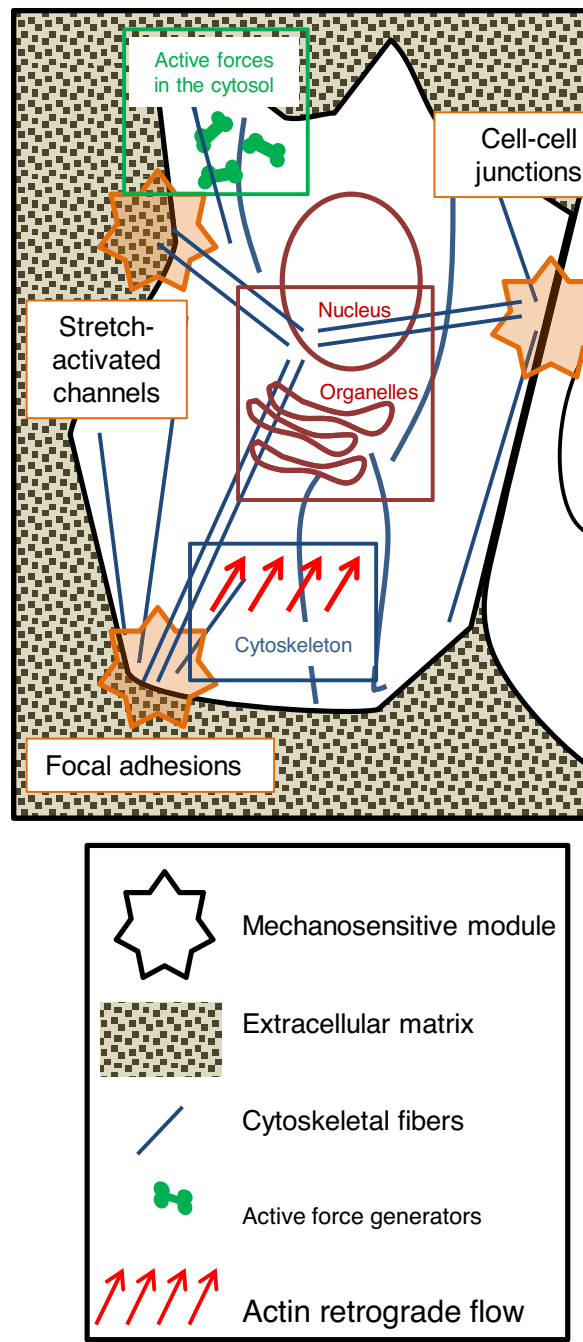

(a) - Active forces in the cytosol
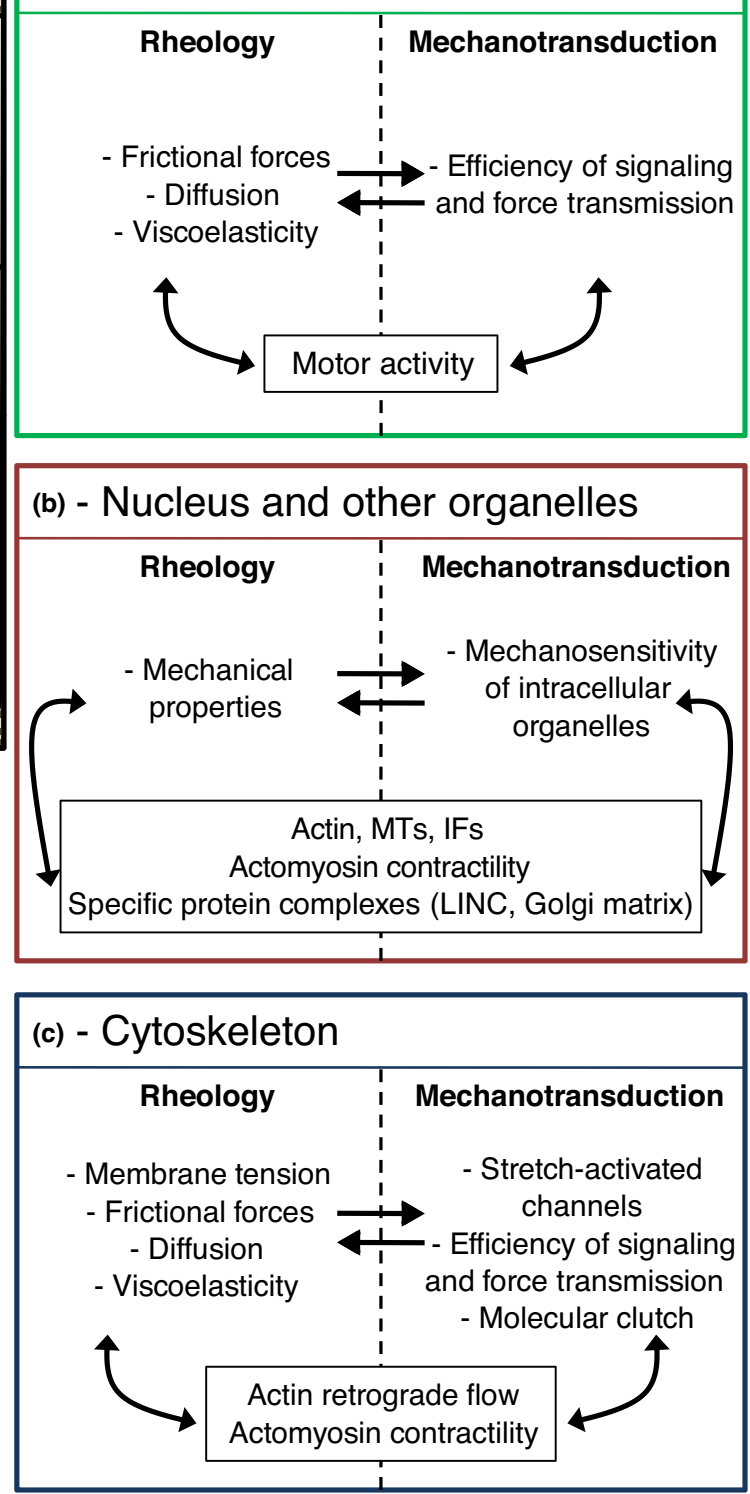

Connections between intracellular rheology and mechanotransduction.

(a) Active forces in the cytosol. A more viscous environment could induce more friction and slow down diffusion-based processes, thus damping potential transmission of forces inside cells. On the other hand, a more elastic medium could enhance sensitivity to deformations and strengthen interactions with cytoskeletal elements. Conversely, biochemical signaling pathways could modify the rheological parameters of the cytoplasm by regulating out-of-equilibrium active processes such actomyosin contractility and/or ATP levels in cells.

(b) Nucleus and other organelles. The mechanical properties of intracellular organelles could play a direct role in their sensitivity to deformations and forces. The three types of cytoskeletal fibers, cell contractility and specific protein complexes are involved simultaneously in organelle rheology and mechanotransduction. For instance chromatin and the lamina network regulate the shape and the mechanical state of the nucleus. The mechanical behavior of the Golgi apparatus involves links between the cytoskeleton, the Golgi matrix and Golgi membranes.

(c) Cytoskeleton. The cytoskeleton is a major actor in both intracellular rheology and mechanotransduction. First, the cytoskeleton regulates membrane tension which controls stretch-activated channels. Second, the effects of the cytoskeleton on frictional forces and on the viscoelastic properties of the cytoplasm could play a key role in the efficiency of mechanotransduction. For instance, an increase in cytoplasm rigidity and in cytoskeleton tension could increase the speed of mechanotransduction through direct force transmission by cytoskeletal fibers. Third, the actin retrograde flow, acto myosin contractility and more generally cytoplasmic flows exert forces on intracellular components and could be implied in mechanosensitive pathways. 
direct force transmission generally occurs on much shorter time scales (subsecond to seconds) than biochemical signaling (minutes) and cell responses such as gene expression (hours). A range of new techniques or combination of techniques is now available with increasing spatial and temporal resolutions, which should allow elucidating further both the physical and the biological mechanisms of intracellular mechanics in the coming years.

\section{Conflict of interest statement}

Nothing declared.

\section{Acknowledgements}

We thank David Pereira and Nathan Lardier for discussions. S.M. is funded by a grant from Sorbonne Université UPMC University Paris 06 (Programme Doctoral 'Interfaces Pour le Vivant'). We apologize to colleagues whose work we could not cite due to space limitations.

\section{References and recommended reading}

Papers of particular interest, published within the period of review, have been highlighted as:

- of special interest

$\bullet$ of outstanding interest

1. Wang N: Review of cellular mechanotransduction. J Phys $D$ Appl Phys 2017, 50233002.

2. Janmey PA, Weitz DA: Dealing with mechanics: mechanisms of force transduction in cells. Trends Biochem Sci 2004, 29:364370.

3. Pegoraro AF, Janmey P, Weitz DA: Mechanical properties of the cytoskeleton and cells. Cold Spring Harb Perspect Biol 2017, 9a022038.

4. Kasza KE, Rowat AC, Liu J, Angelini TE, Brangwynne CP, Koenderink GH, Weitz DA: The cell as a material. Curr Opin Cell Biol 2007, 19:101-107.

5. Stamenović D: Rheological behavior of mammalian cells. Cell Mol Life Sci 2008, 65:3592-3605.

6. Muhamed I, Chowdhury F, Maruthamuthu V: Biophysical tools to study cellular mechanotransduction. Bioengineering 2017 , 4:12.

7. Alibert C, Goud B, Manneville J-B: Are cancer cells really softer than normal cells? Biol Cell 2017, 109.

8. Darling EM, Di Carlo D: High-throughput assessment of cellular mechanical properties. Annu Rev Biomed Eng 2015, 17:35-62.

9. Prabhune M, Rehfeldt F, Schmidt CF: Molecular force sensors to measure stress in cells. J Phys D Appl Phys 2017, 50.

10. Broussard JA, Green KJ: Research techniques made simple: methodology and applications of Förster Resonance Energy Transfer (FRET) microscopy. J Invest Dermatol 2017, 137:e185e191.

11. Gayrard C, Borghi N: FRET-based molecular tension microscopy. Methods 2016, 94:33-42.

12. Hochreiter B, Garcia AP, Schmid JA: Fluorescent proteins as genetically encoded FRET biosensors in life sciences. Sensors (Switzerland) 2015, 15:26281-26314.

13. Guo M, Ehrlicher AJ, Jensen MH, Renz M, Moore JR, Goldman RD, Lippincott-Schwartz J, Mackintosh FC, Weitz DA: Probing the stochastic, motor-driven properties of the cytoplasm using force spectrum microscopy. Cell 2014, 158:822-832.

14. Berret J-F: Local viscoelasticity of living cells measured by rotational magnetic spectroscopy. Nat Commun 2016, 7:10134.
15. Mandal K, Asnacios A, Goud B, Manneville J-B: Mapping - intracellular mechanics on micropatterned substrates. Proc Natl Acad Sci U S A 2016, 113.

Local intracellular mechanical properties are measured in living cells using optical tweezers-based active microrheology combined with adhesive micropatterning. The authors investigate the role of the cytoskeleton, of ATP-dependent active forces and of intracellular membranes in intracellular mechanics. The technique also discriminates between normal and cancer cells.

16. Guo M, Pegoraro AF, Mao A, Zhou EH, Arany PR, Han Y,

- Burnette DT, Jensen MH, Kasza KE, Moore JR et al.: Cell volume change through water efflux impacts cell stiffness and stem cell fate. Proc Natl Acad Sci U S A 2017, 114:E8618-E8627.

This study establishes a link between cell stiffness and cell volume and shows that cell volume influences both cortical and cytoplasmic rigidity.

17. Ahmed WW, Fodor É, Almonacid M, Bussonnier M, Verlhac MH,

- Gov N, Visco P, van Wijland F, Betz T: Active mechanics reveal molecular-scale force kinetics in living oocytes. Biophys $J$ 2018, 114:1667-1679

This article studies the active diffusion of vesicles in mouse oocytes. By combining optical tweezers rheology with theoretical analyses, the authors measure molecular-scale force kineticsin vivo. An average force of $0.4 \mathrm{pN}$ on vesicles due to active diffusion is measured.

18. Rigato A, Miyagi A, Scheuring S, Rico F: High-frequency - microrheology reveals cytoskeleton dynamics in living cells. Nat Phys 2017, 13:771-775.

High-speed AFM microrheology allows probing the viscoelastic response of different cell types after cytoskeletal drug treatments at high frequencies. The technique discriminates between benign and malignant cancer cells.

19. Grasland-Mongrain $P$, Zorgani A, Nakagawa S, Bernard S,

- Paim LG, Fitzharris G, Catheline S, Cloutier G: Ultrafast imaging of cell elasticity with optical microelastography. Proc Natl Acad Sci U S A 2018, 115:861-866 http://dx.doi.org/10.1073/ pnas. 1713395115 .

This article develops a new technique based on shear wave elastography for mapping elasticity in cells at the micrometer scale. The local effect of actin disruption on the shear modulus is measured.

20. Guo M, Ehrlicher AJ, Mahammad S, Fabich $\mathrm{H}$, Jensen $\mathrm{MH}$, Moore JR, Fredberg JJ, Goldman RD, Weitz DA: The role of vimentin intermediate filaments in cortical and cytoplasmic mechanics. Biophys J 2013, 105:1562-1568.

21. Charrier EE, Montel L, Asnacios A, Delort F, Vicart P, Gallet F,

- Batonnet-Pichon $S$, Hénon S: The desmin network is a determinant of the cytoplasmic stiffness of myoblasts. Biol Cell 2018, 110:77-90 http://dx.doi.org/10.1111/boc.201700040.

This article combines several rheology techniques to demonstrate that desmin, an intermediate filament protein expressed in muscle cells, contributes to the cytoplasm viscoelasticity. The results suggest that mutations in desmin could alter the mechanical properties of muscles.

22. Mittasch M, Gross $P$, Nestler M, Fritsch AW, Iserman C, Kar M,

-. Munder M, Voigt A, Alberti S, Grill SW et al.: Non-invasive perturbations of intracellular flow reveal physical principles of cell organization. Nat Cell Biol 2018, 20:344-351.

This article introduces focused-light-induced cytoplasmic streaming (FLUCS) as a technique to study the coupling between cytoplasmic and cortical flows inC. elegans embryos and to measure the effect of energy depletion on the rheology of the cytoplasm in yeast.

23. Almonacid M, Ahmed WW, Bussonnier M, Mailly P, Betz T, Voituriez R, Gov NS, M-H Verlhac: Active diffusion positions the nucleus in mouse oocytes. Nat Cell Biol 2015, 17:470-479.

24. Thorpe SD, Lee DA: Dynamic regulation of nuclear architecture and mechanics - a rheostatic role for the nucleus in tailoring cellular mechanosensitivity. Nucleus 2017, 8:287-300.

25. Isermann $\mathrm{P}$, Lammerding J: Nuclear mechanics and mechanotransduction in health and disease. Curr Biol 2013, 23: R1113-R1121.

26. Pajerowski JD, Dahl KN, Zhong FL, Sammak PJ, Discher DE: Physical plasticity of the nucleus in stem cell differentiation. Proc Natl Acad Sci U S A 2007, 104:15619-15624.

27. Krause M, Te Riet J, Wolf K: Probing the compressibility of tumor cell nuclei by combined atomic force-confocal microscopy. Phys Biol 2013, 10. 
28. Stephens AD, Banigan EJ, Marko JF: Separate roles for chromatin and lamins in nuclear mechanics. Nucleus 2017, 1034:1-6.

29. Beicker K, O’Brien ET, Falvo MR, Superfine R: Vertical light sheet - enhanced side-view Imaging for AFM cell mechanics studies. Sci Rep 2018, 8:1-12.

This article combines side-view intracellular imaging with AFM to visualize subcellular dynamics and correlate force indentation measurements with nuclear deformation. Stiffening is observed when deformation reaches the nuclear envelope.

30. Dahl KN, Engler AJ, Pajerowski JD, Discher DE: Power-law rheology of isolated nuclei with deformation mapping of nuclear substructures. Biophys J 2005, 89:2855-2864.

31. Guilluy C, Osborne LD, Van Landeghem L, Sharek L, Superfine R, Garcia-mata R, Burridge K: Isolated nuclei adapt to force and reveal a mechanotransduction pathway within the nucleus. Nat Cell Biol 2014, 16:376-381.

32. Stephens AD, Banigan EJ, Adam SA, Goldman RD, Marko JF:

- Chromatin and lamin A determine two different mechanical response regimes of the cell nucleus. Mol Biol Cell 2017, 28:1984-1996.

This article studies the mechanical roles of chromatin and lamin A/C in isolated nuclei. Chromatin regulates mechanical responses to small deformations whereas lamin A/C allows protecting nuclear structures against larger stresses.

33. Chan CJ, Li W, Cojoc G, Guck J: Volume transitions of isolated - cell nuclei induced by rapid temperature increase. Biophys $J$ 2017, 112:1063-1076.

This article shows that the volume of isolated nuclei is strongly temperature-dependent. A sudden increase in temperature, but not slow heating, impacts nuclear volume on short timescales.

34. Lee H, Adams WJ, Alford PW, McCain ML, Feinberg AW, Sheehy SP, Goss JA, Parker KK: Cytoskeletal prestress regulates nuclear shape and stiffness in cardiac myocytes. Exp Biol Med 2015, 240:1543-1554.

35. Wang X, Liu H, Zhu M, Cao C, Xu Z, Tsatskis Y, Lau K, Kuok C,

- Filleter T, McNeill H et al.: Mechanical stability of the cell nucleus: roles played by the cytoskeleton in nuclear deformation and strain recovery. J Cell Sci 2018, $131 \mathrm{http}: / / \mathrm{dx}$ doi.org/10.1242/jcs.209627.

This article decouples the viscoelastic parameters of the cell membrane the cytoskeleton and the nucleus by using sharp AFM probe tips and shows that the cytoskeleton lowers the deformability of the nucleus and makes the nucleus sensitive to mechanical stimuli.

36. Cao X, Moeendarbary E, Isermann P, Davidson PM, Wang X,

- $\quad$ Chen MB, Burkart AK, Lammerding J, Kamm RD, Shenoy VB: A chemomechanical model for nuclear morphology and stresses during cell transendothelial migration. Biophys $J$ 2016, 111:1541-1552.

This article develops a chemomechanical model to describe nuclear deformations and to predict nuclear envelope plasticity and rupture. The model includes actin contraction and cytosolic pressure and reproduces nuclear plasticity experimentally observed in lamin A/C-deficient cells.

37. Keeling MC, Flores LR, Dodhy AH, Murray ER, Gavara N:

-. Actomyosin and vimentin cytoskeletal networks regulate nuclear shape, mechanics and chromatin organization. Sci Rep 2017, 7:1-14

Taking advantage of variability in human mesenchymal stem cells, the authors correlate nuclear state and mechanics (nuclear viscoelastic properties, height and volume, chromatin condensation) with the amount of cytoskeletal fibers. They show that the cytoskeleton, in particular stress fibers and intermediate filaments, play a role in the nucleus auxetic properties.

38. Papanikou E, Glick BS: Golgi compartmentation and identity. Curr Opin Cell Biol 2014, 29:74-81.

39. Egea G, Serra-Peinado C: Golgi apparatus: finally mechanics comes to play in the secretory pathway. Curr Biol 2014, 24 : R741-R743.

40. Guet D, Mandal K, Pinot M, Hoffmann J, Abidine Y, Sigaut W, Bardin S, Schauer K, Goud B, Manneville J-B: Mechanical role of actin dynamics in the rheology of the Golgi complex and in Golgi-associated trafficking events. Curr Biol 2014, 24.
41. Charrier EE, Janmey PA: Mechanical properties of intermediate filament proteins. Methods Enzymol 2016, 568:35-57.

42. Block J, Witt H, Candelli A, Peterman EJG, Wuite GJL, Janshoff A

- Köster S: Nonlinear loading-rate-dependent force response of individual vimentin intermediate filaments to applied strain. Phys Rev Lett 2017, 118:048101 http://dx.doi.org/10.1103/ PhysRevLett.118.048101.

This article reports force-strain curves of individual vimentin intermediate filaments and provides a theoretical model to explain the successive unfolding regimes. Force-strain curves are obtained in a large range of strain by combining optical trapping and atomic force microscopy. The study reveals that the force response of vimentin filaments strongly depends on the loading rate.

43. Zhang J, Wang C: Molecular structural mechanics model for the mechanical properties of microtubules. Biomech Model Mechanobiol 2014, 13:1175-1184.

44. Daneshmand F, Amabili M: Coupled oscillations of a protein microtubule immersed in cytoplasm: an orthotropic elastic shell modeling. J Biol Phys 2012, 38:429-448.

45. Nolting JF, Möbius W, Köster S: Mechanics of individual keratin bundles in living cells. Biophys J 2014, 107:2693-2699.

46. Labouesse C, Gabella C, Meister JJ, Vianay B, Verkhovsky AB: Microsurgery-aided in-situ force probing reveals extensibility and viscoelastic properties of individual stress fibers. Sci Rep 2016, 6:1-9.

47. Bosch-Fortea M, Martín-Belmonte F: Mechanosensitive adhesion complexes in epithelial architecture and cancer onset. Curr Opin Cell Biol 2018, 50:42-49.

48. Elosegui-Artola A, Trepat X, Roca-Cusachs P: Control of mechanotransduction by molecular clutch dynamics. Trends Cell Biol 2018, 28:356-367 http://dx.doi.org/10.1016/j. tcb.2018.01.008.

49. Etienne-Manneville S, Lammerding J: Connecting the plasma membrane to the nucleus by intermediate filaments. Mol Biol Cell 2017, 28:695-696.

50. De Pascalis C, Etienne-Manneville S: Single and collective cell migration: the mechanics of adhesions. Mol Biol Cell 2017, 28:1833-1846.

51. Kirby TJ, Lammerding J: Emerging views of the nucleus as a cellular mechanosensor. Nat Cell Biol 2018, 20:373-381.

52. Gauthier NC, Roca-Cusachs P: Mechanosensing at integrinmediated cell-matrix adhesions: from molecular to integrated mechanisms. Curr Opin Cell Biol 2018, 50:20-26.

53. Sarangi BR, Gupta M, Doss BL, Tissot N, Lam F, Mège RM,

- $\quad$ Borghi N, Ladoux B: Coordination between intra- and extracellular forces regulates focal adhesion dynamics. Nano Lett 2017, 17:399-406.

Using micropillar traction force assays, FRET biosensors and super resolution microscopy, the authors show that, at the scale of an individual focal adhesion, molecular tension increases towards the distal end of the focal adhesion.

54. McKenzie AJ, Hicks SR, Svec KV, Naughton H, Edmunds ZL,

- Howe AK: The mechanical microenvironment regulates ovarian cancer cell morphology, migration, and spheroid disaggregation. Sci Rep 2018, 8:1-20.

This article shows in ovarian cancer cells that cell spreading area, density of focal adhesions, cell migration, traction forces, and nuclear localization of YAP all increase with substrate rigidity in an actomyosin/ROCK/FAKdependent manner.

55. Abidine Y, Constantinescu A, Laurent VM, Sundar Rajan V,

- Michel R, Laplaud V, Duperray A, Verdier C: Mechanosensitivity of cancer cells in contact with soft substrates using AFM. Biophys J 2018, 114:1165-1175.

This AFM study links changes in the extracellular environment and loca cellular and intracellular rheological properties in bladder cancer cells. Interestingly, invasive cells stiffen on an endothelial monolayer, while less invasive cells soften on such substrates, suggesting complex links between cell rheology and intracellular mechanotransduction.

56. Georgiadou M, Lilja J, Jacquemet G, Guzmán C, Rafaeva M,

- Alibert C, Yan Y, Sahgal P, Lerche M, Manneville J-B et al.: AMPK 
negatively regulates tensin-dependent integrin activity. J Cell Biol 2017, 216:1107-1121.

This article identifies a novel signaling pathway in cell-substrate adhesion-induced mechanotransduction. Upon loss of AMPK, $\beta 1$-integrin activity increases due to enhanced binding of tensin to $\beta 1$-integrin tails which increases fibrillar adhesion, traction forces and intracellular stiffness.

57. Broussard JA, Getsios S, Green KJ: Desmosome regulation and signaling in disease. Cell Tissue Res 2015, 360:501-512.

58. Broussard JA, Yang R, Huang C, Nathamgari SSP, Beese AM, Godsel LM, Hegazy MH, Lee S, Zhou F, Sniadecki NJ et al.: The desmoplakin-intermediate filament linkage regulates cell mechanics. Mol Biol Cell 2017, 28:3156-3164.

59. McCue S, Noria S, Langille BL: Shear-induced reorganization of endothelial cell cytoskeleton and adhesion complexes. Trends Cardiovasc Med 2004, 14:143-151.

60. Wang D, Zheng W, Xie Y, Gong P, Zhao F, Yuan B, Ma W, Cui Y, Liu W, Sun $Y$ et al.: Tissue-specific mechanical and geometrical control of cell viability and actin cytoskeleton alignment. Sci Rep 2014, 4:1-6.

61. Jahed Z, Shams H, Mehrbod M, Mofrad MRK Mechanotransduction pathways linking the extracellular matrix to the nucleus. Int Rev Cell Mol Biol 2014, 310:171-220.

62. Osmanagic-Myers S, Dechat T, Foisner R: Lamins at the crossroads of mechanosignaling. Genes Dev 2015, 29:225-237.

63. Mellad JA, Warren DT, Shanahan CM: Nesprins LINC the nucleus and cytoskeleton. Curr Opin Cell Biol 2011, 23:47-54.

64. Tapley EC, Starr DA: Connecting the nucleus to the cytoskeleton by SUN-KASH bridges across the nuclear envelope. Curr Opin Cell Biol 2013, 25:57-62.

65. Balikov DA, Brady SK, Ko UH, Shin JH, de Pereda JM, Sonnenberg A, Sung H-J, Lang MJ: The nesprin-cytoskeleton interface probed directly on single nuclei is a mechanically rich system. Nucleus 2017, 8:534-547.

66. Kim D-H, Chambliss AB, Wirtz D: The multi-faceted role of the actin cap in cellular mechanosensation and mechanotransduction. Soft Matter 2013, 9:5516-5523.

67. Makhija E, Jokhun DS, Shivashankar GV: Nuclear deformability and telomere dynamics are regulated by cell geometric constraints. Proc Natl Acad Sci U S A 2016, 113:E32-E40.

68. Kim DH, Wirtz D: Cytoskeletal tension induces the polarized architecture of the nucleus. Biomaterials 2015, 48:161-172.

69. Irianto J, Swift J, Martins RP, McPhail GD, Knight MM, Discher DE, Lee DA: Osmotic challenge drives rapid and reversible chromatin condensation in chondrocytes. Biophys J 2013 104:759-769.

70. Haase $\mathrm{K}$, MacAdangdang JKL, Edrington $\mathrm{CH}$, Cuerrier $\mathrm{CM}$, - Hadjiantoniou S, Harden JL, Skerjanc IS, Pelling AE: Extracellular forces cause the nucleus to deform in a highly controlled anisotropic manner. Sci Rep 2016, 6:1-11.

This article provides evidence that stresses applied to whole cells induce anisotropic and fast deformation of nuclei. Anisotropy is cytoskeleton- dependent (actin and microtubules resist perpendicular strains) and intrinsic (anisotropy is also observed in isolated nuclei).

71. Elosegui-Artola A, Andreu I, Beedle AEM, Lezamiz A, Uroz M,

- Kosmalska AJ, Oria R, Kechagia JZ, Rico-Lastres P, Le Roux AL et al.: Force triggers YAP nuclear entry by regulating transport across nuclear pores. Cell 2017, 171:1397-1410.e14

This article demonstrates that forces exerted via substrate rigidity, AFM, or osmotic shocks are transmitted from the ECM to the nucleus and induce nuclear translocation of YAP, a mechanosensitive transcriptional activator, by regulating transport through nuclear pores.

72. Dupont S, Morsut L, Aragona M, Enzo E, Giulitti S, Cordenonsi M Zanconato F, Le Digabel J, Forcato M, Bicciato S et al.: Role of YAP/TAZ in mechanotransduction. Nature 2011, 474:179-183

73. Ahmed WW, Saif TA: Active transport of vesicles in neurons is modulated by mechanical tension. Sci Rep 2014, 4:1-7.

74. Kim TJ, Joo C, Seong J, Vafabakhsh R, Botvinick EL, Berns MW, Palmer AE, Wang N, Ha T, Jakobsson E et al.: Distinct mechanisms regulating mechanical force-induced $\mathrm{Ca}^{2+}$ signals at the plasma membrane and the ER in human MSCs. eLife 2015, 2015:1-14.

75. Graham DM, Andersen T, Sharek L, Uzer G, Rothenberg K,

- Hoffman BD, Rubin J, Balland M, Bear JE, Burridge K: Enucleated cells reveal differential roles of the nucleus in cell migration, polarity, and mechanotransduction. J Cell Biol 2018, 217:895914.

This article demonstrates that enucleated cells are able to migrate in 1D and $2 \mathrm{D}$. However, migration in 3D environments depends on the nucleus.

76. Tajik A, Zhang Y, Wei F, Sun J, Jia Q, Zhou W, Singh R, Khanna N

- Belmont AS, Wang N: Transcription upregulation via forceinduced direct stretching of chromatin. Nat Mater 2016, 15:1287-1296.

This article demonstrates force-induced transcription. Local stresses applied on the surface of living cells using three-dimensional magnetic twisting cytometry directly transmit to and stretch chromatin. Forceinduced transcription depends on the pre-stressed actin cytoskeleton.

77. Kim JE, Reynolds DS, Zaman MH, Mak M: Characterization of the mechanical properties of cancer cells in 3D matrices in response to collagen concentration and cytoskeletal inhibitors. Integr Biol (United Kingdom) 2018, 10:232-241.

78. Wang P, Dreger M, Madrazo E, Williams CJ, Samaniego R,

- Hodson NW, Monroy F, Baena E, Sánchez-Mateos P: WDR5 modulates cell motility and morphology and controls nuclear changes induced by a 3D environment. Proc Natl Acad Sci U S A 2018, 115:8581-8586 http://dx.doi.org/10.1073/ pnas. 1719405115

This article demonstrates that epigenetic changes in 3D environments are accompanied by changes in nuclear mechanics. Nuclei from cells cultured in 3D are softer than nuclei from cells cultured in 2D, revealing a potential link between mechanosensing and nuclear rheology.

79. Peglion F, Llense F, Etienne-Manneville S: Adherens junction treadmilling during collective migration. Nat Cell Biol 2014, 16:639-651.

80. Jankowska KI, Williamson EK, Roy NH, Blumenthal D, Chandra V, Baumgart T, Burkhardt JK: Integrins modulate T cell receptor signaling by constraining actin flow at the immunological synapse. Front Immunol 2018, 9:1-19. 